\title{
THE REPTATING ROPE MODEL: VISCOMETRIC FUNCTIONS FOR CONCENTRATED POLYMER SOLUTIONS AND MELTS IN SHEAR FLOW
}

\section{BERNARD J. GEURTS}

Center for Theoretical Physics, Twente University, P.O. Box 217, Enschede 7500 AE (The Netherlands)

(Received October 10, 1987; in revised form June 8, 1988)

\section{Summary}

The viscometric functions for shear flow as predicted by the inextensible reptating rope model have been analysed numerically and analytically. The results obtained are compared with the predictions of the Curtiss-Bird theory. It is shown that if the correlation length of the rope is small as compared to its contour length significant deviations from the Curtiss-Bird theory are obtained. Results are presented for: (a) the onset of shear flow, (b) steady state shear flow and (c) small amplitude oscillatory shear flow.

\section{Introduction}

Recently a new analysis was given of the elastic reptating rope model [1-3]. The explicit constitutive equation for the inextensible reptating rope model was derived [4]. It was shown that the constitutive equation as obtained by Curtiss and Bird [5-10], with 'link tension coefficient' equal to $1 / 2$, resulted from the inextensible reptating rope model as a special case, by taking the correlation length equal to the contour length of the rope. In the present paper we analyse numerically and analytically the viscometric functions of concentrated polymer solutions and melts in shear flow as predicted by the inextensible reptating rope model. By comparing these results with the predictions of the Curtiss-Bird theory we also show the contribution of the new correlation related term in our constitutive equation [4] which appeared in the theory as a result of the use of the two segment distribution function derived in Ref. 1. 
In the reptating rope model the molecule is represented by a continuous space curve of contour length $L$ ('rope'). This rope is supposed to be confined in a tube of the same shape and about the same cross-section. This tube represents, in a mean-field sense [11], the topological constraints imposed on the motion of the molecule by the other molecules in the system. The molecule considered has a correlation length $a$, which implies that any configuration of the molecule can be represented by $N \equiv L / a$ segments of length $a$. The tube segments associated with this are considered to move affinely along with the macroscopic flow, maintaining the same cross-sectional area. Our approach is thus very similar to that of Doi and Edwards [12-15]. We have added a hydrodynamic force density resulting from the relative motion of the rope with respect to the tube. This interaction with the medium is of vital importance for the determination of the stress tensor. The details of the derivation of the constitutive equation may be found in Ref. 4 .

In Section 2 we describe the constitutive equation for the inextensible reptating rope model and consider the kinematic tensors for shear flow. Then, in Section 3 we derive explicit formulae for the viscometric functions for the onset of shear flow and present a numerical study of these functions. The steady state shear flow is treated both analytically (for small shear rates) as well as numerically in Section 4 showing large deviations from the Curtiss-Bird theory at high shear rates. Finally in Section 5 we analyse small amplitude oscillatory shear flow analytically.

\section{The constitutive equation and kinematic tensors for shear flow}

In this section we describe the constitutive equation for the inextensible reptating rope model as derived in Ref. 4 . Then we consider the kinematic tensors for general shear flow.

The polymer contribution to the stress tensor $\boldsymbol{T}(t)$ was derived in Ref. 4 and can be written as

$$
\begin{aligned}
\boldsymbol{T}(t)= & n N k T\left[\int_{-\infty}^{t} \mathrm{~d} t^{\prime} \mu\left(t-t^{\prime}\right) \Omega\left(t, t^{\prime}\right)+\int_{-\infty}^{t} \mathrm{~d} t^{\prime} A\left(t-t^{\prime} ; \Delta\right) \boldsymbol{J}\left(t, t^{\prime}\right)\right. \\
& \left.+\int_{-\infty}^{t} \mathrm{~d} t^{\prime} \int_{-\infty}^{t} \mathrm{~d} t^{\prime \prime} B\left(t-t^{\prime}, t-t^{\prime \prime} ; \Delta\right) \boldsymbol{D}(t): \Omega\left(t, t^{\prime}\right) \Omega\left(t, t^{\prime \prime}\right)\right],
\end{aligned}
$$

where $\Delta \equiv a / L$ is the reduced correlation length, $k$ is Boltzmann's constant, $T$ the temperature and $n$ the number density of polymers. The tensors $\Omega$ and $\boldsymbol{J}$ are given by

$$
\begin{aligned}
& \boldsymbol{\Omega}\left(t, t^{\prime}\right)=\left\langle\boldsymbol{g}\left(t, t^{\prime}, \boldsymbol{e}\right) \boldsymbol{g}\left(t, t^{\prime}, \boldsymbol{e}\right)\right\rangle_{0}, \\
& \boldsymbol{J}\left(t, t^{\prime}\right)=\boldsymbol{D}(t):\left\langle\boldsymbol{g}\left(t, t^{\prime}, \boldsymbol{e}\right) \boldsymbol{g}\left(t, t^{\prime}, \boldsymbol{e}\right) \boldsymbol{g}\left(t, t^{\prime}, \boldsymbol{e}\right) \boldsymbol{g}\left(t, t^{\prime}, \boldsymbol{e}\right)\right\rangle_{0},
\end{aligned}
$$


where

$g\left(t, t^{\prime}, e\right)=\frac{F_{t^{\prime}}(t) \cdot e}{\left|F_{t^{\prime}}(t) \cdot e\right|}$

and

$\langle\cdots\rangle_{0}=\frac{1}{4 \pi} \int_{0}^{\pi} \mathrm{d} \vartheta \int_{0}^{2 \pi} \mathrm{d} \varphi \sin (\vartheta)(\cdots)$.

Here $e(=e(\vartheta, \varphi))$ is a unit vector with uniform orientational distribution function, consistent with the reptation model that pictures endsegments to be created in arbitrary directions with equal probability. The tensor $F_{t^{\prime}}(t) \equiv$ $\partial \boldsymbol{x}(t) / \partial \boldsymbol{x}\left(t^{\prime}\right)$ is the macroscopic deformation gradient and $\boldsymbol{D}$ is the macroscopic rate of strain tensor. The weighting functions $\mu, A$ and $B$ can be expressed as

$\mu(x)=\frac{8}{\pi^{2} \tau_{d}} \sum_{m=0}^{\infty} \exp \left(-m^{2} \frac{x}{\tau_{d}}\right)$

$A(x ; \Delta)=\frac{8}{\pi^{2}} \sum_{m=0}^{\infty} \frac{\alpha_{m}(\Delta)}{m^{2}} \exp \left(-m^{2} \frac{x}{\tau_{d}}\right)$,

$B(x, y ; \Delta)=\frac{16}{\tau_{d}} \sum_{m=0}^{\infty} \sum_{p=0}^{\infty} m p \beta_{m, p}(\Delta) \exp \left(-m^{2} \frac{x}{\tau_{d}}\right) \exp \left(-p^{2} \frac{y}{\tau_{d}}\right)$,

where $\tau_{d} \equiv L^{2} / \pi^{2} D$ is the 'disengagement time' first introduced by Doi and Edwards ( $D$ is the diffusion coefficient characterizing the reptative diffusion of segments along the rope, it scales as $N^{-1}$ as $\left.N \gg 1[11,16]\right)$. The prime on the summations indicates that we only sum over odd integers $m$ and $p$.

The combinatorial factors $\alpha_{m}(\Delta)$ and $\beta_{m, p}(\Delta)$ are given by

$$
\begin{aligned}
\alpha_{m}(\Delta)= & {[2 \Delta-1]+[1-\Delta] \cos (m \pi \Delta)+\frac{3}{m \pi} \sin (m \pi \Delta) } \\
\beta_{m, m}(\Delta)= & {[1-\Delta] \frac{\sin (m \pi \Delta)}{2 m^{3} \pi^{3}}+[1-\Delta]^{2} \frac{\cos (m \pi \Delta)}{2 m^{2} \pi^{2}} } \\
& -[1-\Delta]^{3} \frac{\sin (m \pi \Delta)}{6 m \pi} \\
\beta_{m . p}(\Delta)= & \frac{16 m p}{\pi^{4}\left(m^{2}-p^{2}\right)^{3}}[\cos (p \pi \Delta)-\cos (m \pi \Delta)] \\
& +4[1-\Delta] \frac{m \sin (p \pi \Delta)+p \sin (m \pi \Delta)}{\pi^{3}\left(m^{2}-p^{2}\right)^{2}}
\end{aligned}
$$


We notice that the expression for the stress tensor $\boldsymbol{T}(t)$ in (2.1) contains three terms. The first is exactly equal to the constitutive equation as described by Doi and Edwards. The second term is of the same form as the extra term first derived by Curtiss and Bird, with 'link-tension coefficient' equal to $1 / 2$. However the weighting function $A$ differs slightly from their corresponding weighting function [4]. It is smaller than the weighting function as derived by Curtiss and Bird, i.e. $A(x ; \Delta)<A(x ; 1)$ if $\Delta<1$ and coincides with theirs if $\Delta=1$. The third term is essentially new and resembles a quadratic form of the Doi and Edwards term. It becomes relevant if $\Delta \ll 1$ since $A \rightarrow 0$ in this case. Hence, we expect that as $\Delta$ is close to 1 the viscometric functions differ only slightly from the predictions obtained from the Curtiss-Bird theory. These deviations will become significant only as $\Delta$ is small enough. This is confirmed by the numerical results described below in Sections 3 and 4.

We now derive the kinematic tensors for general shear flow. The macroscopic velocity field $v(r, t)$ can be written as

$v_{x}=0 ; \quad v_{y}=\dot{\gamma}(t) z ; \quad v_{z}=0$,

where $\dot{\gamma}(t)$ is the shear rate at time $t$. If we define

$\Gamma\left(t, t^{\prime}\right)=\int_{t^{\prime}}^{t} \mathrm{~d} t^{\prime \prime} \dot{\gamma}\left(t^{\prime \prime}\right)$

then the relevant components of the tensors $\Omega$ and $J$ are given by [8]

$$
\begin{aligned}
& \Omega_{y z}=\frac{1}{2 \Gamma} \int_{0}^{1} \mathrm{~d} x\left[1+h^{-1}(\Gamma, x)\left(\Gamma^{2} x^{2}-1\right)\right], \\
& \Omega_{y y}-\Omega_{z z}=\Gamma \Omega_{y z}, \\
& \Omega_{z z}-\Omega_{x x}=-\frac{1}{2} \int_{0}^{1} \mathrm{~d} x\left[1-h^{-1}(\Gamma, x)\left(\left(\Gamma^{2}+6\right) x^{2}-1\right)\right],
\end{aligned}
$$

with

$h(\Gamma, x)=\left[\left(\Gamma^{4}+4 \Gamma^{2}\right) x^{4}-2 \Gamma^{2} x^{2}+1\right]^{1 / 2}$

and

$$
\begin{aligned}
J_{y z}= & \frac{\dot{\gamma}}{4 \Gamma^{2}} \int_{0}^{1} \mathrm{~d} x\left[1+h^{-3}(\Gamma, x)\right. \\
& \left.\times\left[\left(3 \Gamma^{6}+8 \Gamma^{4}\right) x^{6}-\left(7 \Gamma^{4}+8 \Gamma^{2}\right) x^{4}+5 \Gamma^{2} x^{2}-1\right]\right],
\end{aligned}
$$




$$
\begin{aligned}
J_{y y}-J_{z z}= & \frac{\dot{\gamma}}{4 \Gamma} \int_{0}^{1} \mathrm{~d} x\left[\frac{3+2 \Gamma^{2}}{1+\Gamma^{2}}+h^{-3}(\Gamma, x)\left[\left(2 \Gamma^{6}+2 \Gamma^{4}-8 \Gamma^{2}\right) x^{6}\right.\right. \\
& \left.\left.-\left(7 \Gamma^{4}+8 \Gamma^{2}\right) x^{4}+\left(6 \Gamma^{2}-6\right) x^{2}-1\right]\right], \\
J_{z z}-J_{x x}= & -\frac{\dot{\gamma}}{4} \int_{0}^{1} \mathrm{~d} x\left[h^{-1}(\Gamma, x) 2 \Gamma x^{2}-h^{-3}(\Gamma, x)\right. \\
& \left.\times\left[\left(2 \Gamma^{3}+4 \Gamma\right) x^{4}-2 \Gamma x^{2}\right]\left[\left(\Gamma^{2}+6\right) x^{2}-1\right]\right] \\
= & -\frac{1}{2} \dot{\gamma} \frac{\mathrm{d}}{\mathrm{d} \Gamma}\left(\Omega_{z z}-\Omega_{x x}\right) .
\end{aligned}
$$

If one specifies the shear rate $\dot{\gamma}(t)$ then the stress tensor for the corresponding shear flow can be obtained from insertion of the kinematic tensors in eqn. (2.1). This yields a complicated expression that can only be treated numerically.

For later use we list the behavior of these components as $\Gamma \ll 1$. As given by Curtiss and Bird [8] and Doi and Edwards [14] one has

$$
\begin{aligned}
& \Omega_{y z}=\frac{1}{5} \Gamma-\frac{1}{21} \Gamma^{3}+\ldots, \\
& J_{y z}=\dot{\gamma}\left(\frac{1}{15}+\frac{1}{35} \Gamma^{2}+\ldots\right), \\
& \Omega_{z z}-\Omega_{x x}=-\frac{2}{35} \Gamma^{2}+\frac{4}{231} \Gamma^{4}+\ldots, \\
& J_{y y}-J_{z z}=\dot{\gamma}\left(\frac{1}{21} \Gamma^{3}-\frac{10}{429} \Gamma^{5}+\ldots\right) .
\end{aligned}
$$

In the next section we derive explicit formulae for the viscometric functions for the onset of shear flow and present numerical results. The results for steady shear flow will be discussed in Section 4 .

\section{Stress growth after sudden onset of shear flow}

In this section we define the viscometric functions for the onset of shear flow and derive explicit formulae from which these functions can be obtained. Finally we present results obtained from a systematic numerical study of these functions.

The shear rate $\dot{\gamma}(t)$ for the onset of shear flow at $t=0$ can be written as

$$
\begin{array}{rlrl}
\dot{\gamma}(t) & =0 ; & & t<0, \\
& =\dot{\gamma}_{0} ; \quad & t \geqslant 0,
\end{array}
$$

where $\dot{\gamma}_{0}$ is a constant shear rate. This implies that

$$
\begin{aligned}
\Gamma\left(t, t^{\prime}\right) & =\dot{\gamma}_{0}\left(t-t^{\prime}\right) ; & & 0 \leqslant t^{\prime} \leqslant t, \\
& =\dot{\gamma}_{0} t & & t^{\prime} \leqslant 0 .
\end{aligned}
$$


The material functions completely specify the stress tensor and are defined by

$\eta^{+}\left(t ; \dot{\gamma}_{0}\right) \equiv \frac{T_{y z}(t)}{\dot{\gamma}_{0}}$

$\Psi_{1}^{+}\left(t ; \dot{\gamma}_{0}\right) \equiv \frac{T_{y y}(t)-T_{z z}(t)}{\dot{\gamma}_{0}^{2}}$,

$\Psi_{2}^{+}\left(t ; \dot{\gamma}_{0}\right) \equiv \frac{T_{z z}(t)-T_{x x}(t)}{\dot{\gamma}_{0}^{2}}$.

Here $\eta^{+}$is the shear growth viscosity and $\Psi_{1}^{+}, \Psi_{2}^{+}$are the shear growth first and second normal stress coefficients respectively. From a measurement of these functions the stress tensor can be determined. We will now derive an explicit expression for $\eta^{+}$. The other two material functions are given by similar expressions, provided one makes suitable substitution, which will be specified below.

Inserting $(3.3,4)$ in $(2.14)$ and $(2.18)$, and correctly splitting up the integrations involved in (2.1), one obtains after some manipulation

$$
\begin{aligned}
& \frac{\eta^{+}\left(t ; \dot{\gamma}_{0}\right)}{n N k T \tau_{d}} \\
& =\frac{1}{\dot{\gamma}_{0} \tau_{d}}\left[\Omega_{y z}\left(\dot{\gamma}_{0} t\right) \sum_{m=0}^{\infty} \frac{8}{\pi^{2} m^{2}} e^{-m^{2} t / \tau_{d}}\right. \\
& \quad+\int_{0}^{t / \tau_{d}} \mathrm{~d} z^{\prime} \sum_{m=0}^{\infty} \frac{8}{\pi^{2}} e^{-m^{2} / z^{\prime}} \Omega_{y z}\left(\dot{\gamma}_{0} \tau_{d} z^{\prime}\right) \\
& \quad+\tau_{d} J_{y z}\left(\dot{\gamma}_{0} t\right) \sum_{m=0}^{\infty} \frac{8 \alpha_{m}(\Delta)}{\pi^{2} m^{4}} e^{-m^{2} t / \tau_{d}} \\
& \quad+\int_{0}^{t / \tau_{d}} \mathrm{~d} z^{\prime} A\left(\tau_{d} z^{\prime} ; \Delta\right) \tau_{d} J_{y z}\left(\dot{\gamma}_{0} \tau_{d} z^{\prime}\right) \\
& \quad+\int_{t / \tau_{d}}^{\infty} \mathrm{d} z^{\prime}\left[\int_{t / \tau_{d}}^{\infty} \mathrm{d} z^{\prime \prime} \tau_{d} B\left(\tau_{d} z^{\prime}, \tau_{d} z^{\prime \prime} ; \Delta\right) \dot{\gamma}_{0} \tau_{d} \Omega_{y z}\left(\dot{\gamma}_{0} t\right) \Omega_{y z}\left(\dot{\gamma}_{0} t\right)\right. \\
& \left.\quad+\int_{0}^{t / \tau_{d}} \mathrm{~d} z^{\prime \prime} \tau_{d} B\left(\tau_{d} z^{\prime}, \tau_{d} z^{\prime \prime} ; \Delta\right) \dot{\gamma}_{0} \tau_{d} \Omega_{y z}\left(\dot{\gamma}_{0} t\right) \Omega_{y z}\left(\dot{\gamma}_{0} \tau_{d} z^{\prime \prime}\right)\right] \\
& \quad+\int_{0}^{t / \tau_{d}} \mathrm{~d} z^{\prime}\left[\int_{t / \tau_{d}}^{\infty} \mathrm{d} z^{\prime \prime} \tau_{d} B\left(\tau_{d} z^{\prime}, \tau_{d} z^{\prime \prime} ; \Delta\right) \dot{\gamma}_{0} \tau_{d} \Omega_{y z}\left(\dot{\gamma}_{0} \tau_{d} z^{\prime}\right) \Omega_{y z}\left(\dot{\gamma}_{0} t\right)\right. \\
& \left.\left.\quad+\int_{0}^{t / \tau_{d}} \mathrm{~d} z^{\prime \prime} \tau_{d} B\left(\tau_{d} z^{\prime}, \tau_{d} z^{\prime \prime} ; \Delta\right) \dot{\gamma}_{0} \tau_{d} \Omega_{y z}\left(\dot{\gamma}_{0} \tau_{d} z^{\prime}\right) \Omega_{y z}\left(\dot{\gamma}_{0} \tau_{d} z^{\prime \prime}\right)\right]\right]
\end{aligned}
$$


This is a complicated expression which we will rewrite in a form that is more suitable for numerical treatment. As may be verified, $\eta^{+}$is a function of $\dot{\gamma}_{0} \tau_{d}, t / \tau_{d}$ and $\Delta$ only. The first two terms constitute the contribution of the Doi and Edwards theory, the third and fourth terms give the Curtiss-Birdlike contributions and the last 4 terms yield the contribution due to the new correlation-related term in the constitutive equation. By performing some partial integrations and rearrangements of these terms, which will be explained below, one arrives at a more compact and numerically more convenient representation of (3.8).

If we consider the first two terms, and perform a partial integration in the second, these two terms can be combined to give

$\dot{\gamma}_{0} \tau_{d} \int_{0}^{t / \tau_{d}} \mathrm{~d} z^{\prime} \Omega_{y z}^{\prime}\left(\dot{\gamma}_{0} \tau_{d} z^{\prime}\right) \sum_{m=0}^{\infty} \frac{8}{\pi^{2} m^{2}} e^{-m^{2} z^{\prime}}$,

where use has been made of (2.21) and $\Omega_{y z}^{\prime}(\Gamma)=\mathrm{d} / \mathrm{d} \Gamma\left(\Omega_{y z}(\Gamma)\right)$. Performing a partial integration in the fourth term and combining this with the third gives

$\tau_{d} J_{y z}(0) \sum_{m=0}^{\infty} \frac{8 \alpha_{m}(\Delta)}{\pi^{2} m^{4}}+\dot{\gamma}_{0} \tau_{d} \int_{0}^{t / \tau_{d}} \mathrm{~d} z^{\prime} \tau_{d} J_{y z}^{\prime}\left(\dot{\gamma}_{0} \tau_{d} z^{\prime}\right) \sum_{m=0}^{\infty} \frac{8 \alpha_{m}(\Delta)}{\pi^{2} m^{4}} e^{m^{2} / z^{\prime}}$.

The prime on $J_{y z}$ again denotes differentiation with respect to its argument. A straightforward calculation yields

$\sum_{m=0}^{\infty} \frac{8 \alpha_{m}(\Delta)}{\pi^{2} m^{4}}=\frac{\pi^{2}}{12}\left(4 \Delta-6 \Delta^{2}+4 \Delta^{3}-\Delta^{4}\right)$

showing that the first term in (3.10) gives

$\dot{\gamma}_{0} \tau_{d} \frac{\pi^{2}}{180}\left(4 \Delta-6 \Delta^{2}+4 \Delta^{3}-\Delta^{4}\right)$.

This result will be used later on in analysing the small time behavior of $\eta^{+}$ and the linear viscoelasticity.

We now turn to the last four terms in (3.8). The fifth term in (3.8) can be evaluated analytically. Moreover, the integration over $z^{\prime}$ in the sixth and over $z^{\prime \prime}$ in the seventh term can be performed. In total, this yields explicitly, for the last four terms between brackets in (3.8)

$$
\begin{aligned}
& \dot{\gamma}_{0} \tau_{d}\left[\Omega_{y z}\left(\dot{\gamma}_{0} t\right) \Omega_{y z}\left(\dot{\gamma}_{0} t\right) \sum_{m=0}^{\infty} \sum_{p=0}^{\infty} \frac{16 \beta_{m, p}(\Delta)}{m p} \exp \left(-\left(m^{2}+p^{2}\right) \frac{t}{\tau_{d}}\right)\right. \\
& \quad+\Omega_{y z}\left(\dot{\gamma}_{0} t\right) \int_{0}^{t / \tau_{d}} \mathrm{~d} z^{\prime \prime} \Omega_{y z}\left(\dot{\gamma}_{0} \tau_{d} z^{\prime \prime}\right) \sum_{m=0}^{\infty} \sum_{p=0}^{\infty} \frac{16 p \beta_{m, p}(\Delta)}{m} e^{-m^{2} t / \tau_{d}^{\prime}} e^{-p^{2} z^{\prime \prime}}
\end{aligned}
$$




$$
\begin{aligned}
& +\Omega_{y z}\left(\dot{\gamma}_{0} t\right) \int_{0}^{t / \tau_{d}} \mathrm{~d} z^{\prime} \Omega_{y z}\left(\dot{\gamma}_{0} \tau_{d} z^{\prime}\right) \sum_{m=0}^{\infty} \sum_{p=0}^{\infty} \frac{16 m \beta_{m, p}(\Delta)}{p} e^{-m^{2} z^{\prime}} e^{-p^{2} t / \tau_{d}} \\
& +\int_{0}^{t / \tau_{d}} \mathrm{~d} z^{\prime} \int_{0}^{t / \tau_{d}} \mathrm{~d} z^{\prime \prime} \Omega_{y z}\left(\dot{\gamma}_{0} \tau_{d} z^{\prime}\right) \Omega_{y z}\left(\dot{\gamma}_{0} \tau_{d} z^{\prime \prime}\right) \\
& \left.\times \sum_{m=0}^{\infty} \sum_{p=0}^{\infty} 16 m p \beta_{m, p}(\Delta) e^{-m^{2} z^{\prime}} e^{-p^{2} z^{\prime \prime}}\right]
\end{aligned}
$$

In view of the expressions $(2.10,11)$ for $\beta_{m, p}(\Delta)$, one notices either slow convergence or divergence of the series in the second, third and fourth terms in (3.13) for small values of $z^{\prime}$ and $z^{\prime \prime}$. This produces serious problems if one wants to evaluate this expression numerically. However, if we perform partial integrations with respect to $z^{\prime \prime}$ in the second, $z^{\prime}$ in the third and $z^{\prime}$ and $z^{\prime \prime}$ in the fourth term in (3.13) one obtains a great simplification yielding in total

$$
\begin{aligned}
& \left(\dot{\gamma}_{0} \tau_{d}\right)^{3} \int_{0}^{t / \tau_{d}} \mathrm{~d} z^{\prime} \int_{0}^{t / \tau_{d}} \mathrm{~d} z^{\prime \prime} \Omega_{y z}^{\prime}\left(\dot{\gamma}_{0} \tau_{d} z^{\prime}\right) \Omega_{y z}^{\prime}\left(\dot{\gamma}_{0} \tau_{d} z^{\prime \prime}\right) \\
& \times \sum_{m=0}^{\infty} \sum_{p=0}^{\infty} \frac{16 \beta_{m, p}(\Delta)}{m p} e^{m^{2} z^{\prime}} e^{-p^{2} z^{\prime \prime}} .
\end{aligned}
$$

After these manipulations we can rewrite (3.8) as

$$
\begin{aligned}
& \frac{\eta^{+}\left(t ; \dot{\gamma}_{0}\right)}{n N k T \tau_{d}}=\frac{1}{\underline{\dot{\gamma}_{0} \tau_{d}}}\left[\dot{\gamma}_{0} \tau_{d} \int_{0}^{t / \tau d} \mathrm{~d} z^{\prime} \Omega_{y z}^{\prime}\left(\dot{\gamma}_{0} \tau_{d} z^{\prime}\right) \sum_{m=0}^{\infty} \frac{8}{\pi^{2} m^{2}} e^{-m^{2} z^{\prime}}\right. \\
& \quad+\tau_{d} \underline{J_{y z}}(0) \sum_{m=0}^{\infty} \frac{8 \alpha_{m}(\Delta)}{\pi^{2} m^{4}}+\dot{\gamma}_{0} \tau_{d} \int_{0}^{t / \tau_{d}} \mathrm{~d} z^{\prime} \tau_{d} \underline{J_{y z}^{\prime}}\left(\dot{\gamma}_{0} \tau_{d} z^{\prime}\right) \sum_{m=0}^{\infty} \frac{8 \alpha_{m}(\Delta)}{\pi^{2} m^{4}} e^{-m^{2} z^{\prime}} \\
& \quad+\left(\dot{\gamma}_{0} \tau_{d}\right)^{3} \int_{0}^{t / \tau_{d}} \mathrm{~d} z^{\prime} \int_{0}^{t / \tau_{d}} \mathrm{~d} z^{\prime \prime} \Omega_{y z}^{\prime}\left(\dot{\gamma}_{0} \tau_{d} z^{\prime}\right) \underline{\Omega_{y z}^{\prime}}\left(\dot{\gamma}_{0} \tau_{d} z^{\prime \prime}\right) \\
& \left.\quad \times \sum_{m=0}^{\infty} \sum_{p=0}^{\infty} \frac{16 \beta_{m, p}(\Delta)}{m p} e^{-m^{2} z^{\prime}} e^{-p^{2} z^{\prime \prime}}\right]
\end{aligned}
$$

The derivation for the other material functions is completely analogous. $\Psi_{1}^{+}$and $\Psi_{2}^{+}$follow from similar expressions provided one substitutes $\tau_{d}^{2}$ for $\tau_{d}$ on the left-hand side in (3.15), and the underlined $\dot{\gamma}_{0} \tau_{d}$ by $\left(\dot{\gamma}_{0} \tau_{d}\right)^{2}$ on the right-hand side. Further, one should replace the underlined $y z$-components of $\Omega$ and $J$ in (3.15) by the appropriate combination of components consistent with the definitions $(3.6,7)$. Notice that after these manipulations only rapidly converging series appear on the right hand side of (3.15) which makes a numerical treatment of $\eta^{+}$easier to perform. 


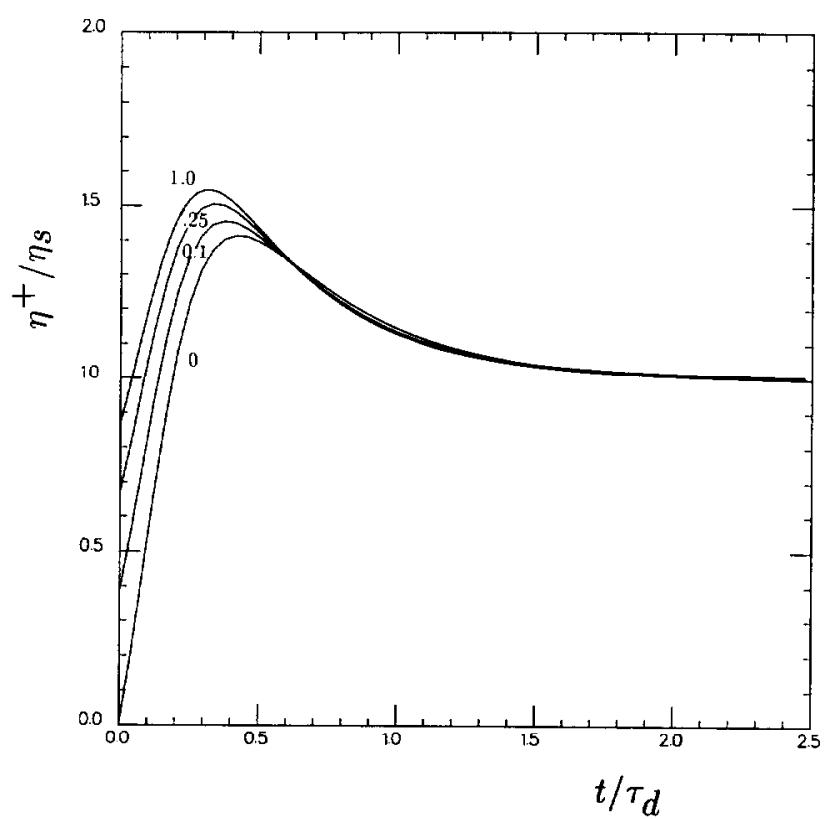

Fig. 1. Shear growth viscosity $\eta^{+}$normalized to the stationary value $\eta_{s}$ as a function of $t / \tau_{d}$ at $\dot{\gamma}_{0} \tau_{d}=5$. The index on the curves corresponds to the value of the reduced correlation length used.

The second term in (3.15) implies that $\eta^{+}$shows a discontinuity as $t \rightarrow 0$ with a jump given by

$$
\lim _{t \downarrow 0} \frac{\eta^{+}\left(t ; \dot{\gamma}_{0}\right)}{n K k T \tau_{d l}}=\frac{\pi^{2}}{180}\left(4 \Delta-6 \Delta^{2}+4 \Delta^{3}-\Delta^{4}\right)
$$

as can be inferred from (3.15), taking into account eqn. (3.12). Notice that this jump tends to zero as $\Delta$ tends to zero. Since the corresponding term for the other two material functions $\Psi_{1}^{+}$and $\Psi_{2}^{+}$is zero in view of $(2.23,24)$, these functions do not show this discontinuity.

In Fig. 1 we plotted $\eta^{+} / \eta_{s}$ as a function of $t / \tau_{d}$. We used $\dot{\gamma}_{0} \tau_{d}=5$ and varied the reduced correlation length $\Delta$. The quantity $\eta_{s}$ denotes the stationary value of $\eta^{+}$corresponding to the prescribed values of $\dot{\gamma}_{0} \tau_{d}$ and $\Delta$. Notice the decreasing discontinuity in the $t \rightarrow 0$ limit as $\Delta$ is decreased, which is in agreement with (3.16). In order to observe significant deviations from the Curtiss-Bird theory $(\Delta=1)$, the reduced correlation length has to be quite small, as mentioned in the previous section. The time at which $\eta^{+} / \eta_{s}$ has a maximum increases and the maximal value slightly decreases as $\Delta$ decreases. The value for $t / \tau_{d}$ at which the maximum is attained roughly doubles as $\Delta$ is varied from 1 to 0 . We also studied variations in $\dot{\gamma}_{0} \tau_{d}$ and 


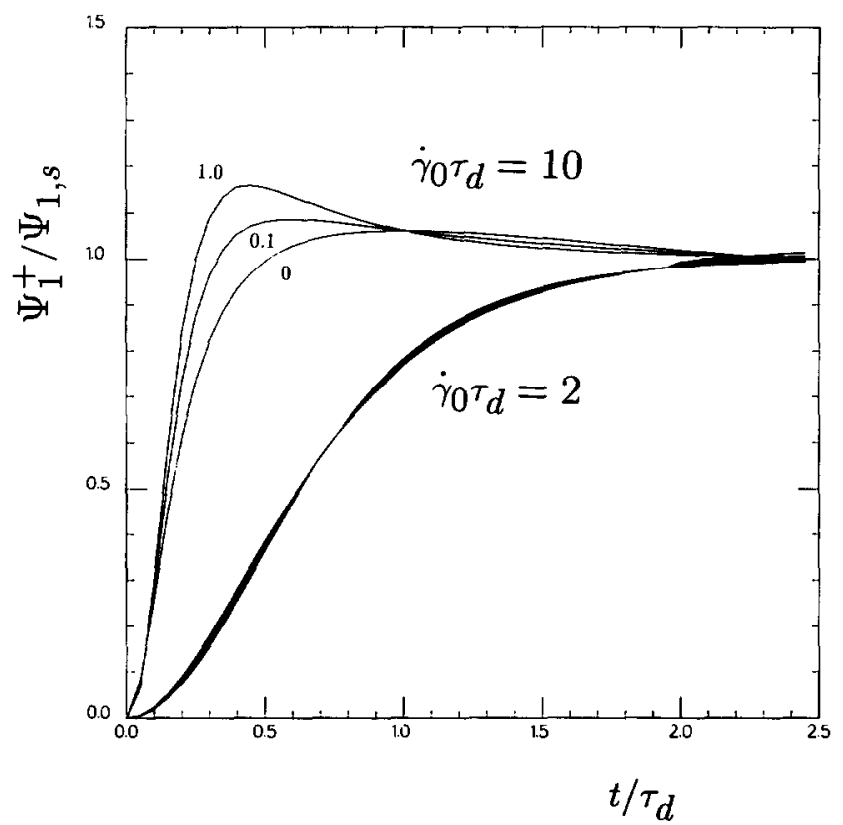

Fig. 2. Shear growth first normal stress coefficient $\Psi_{1}^{+}$normalized to the stationary value $\Psi_{1, s}$ as a function of $t / \tau_{d}$ at $\dot{\gamma}_{0} \tau_{d}=2$ and 10. The corresponding values of $\Delta$ are indicated on the curves.

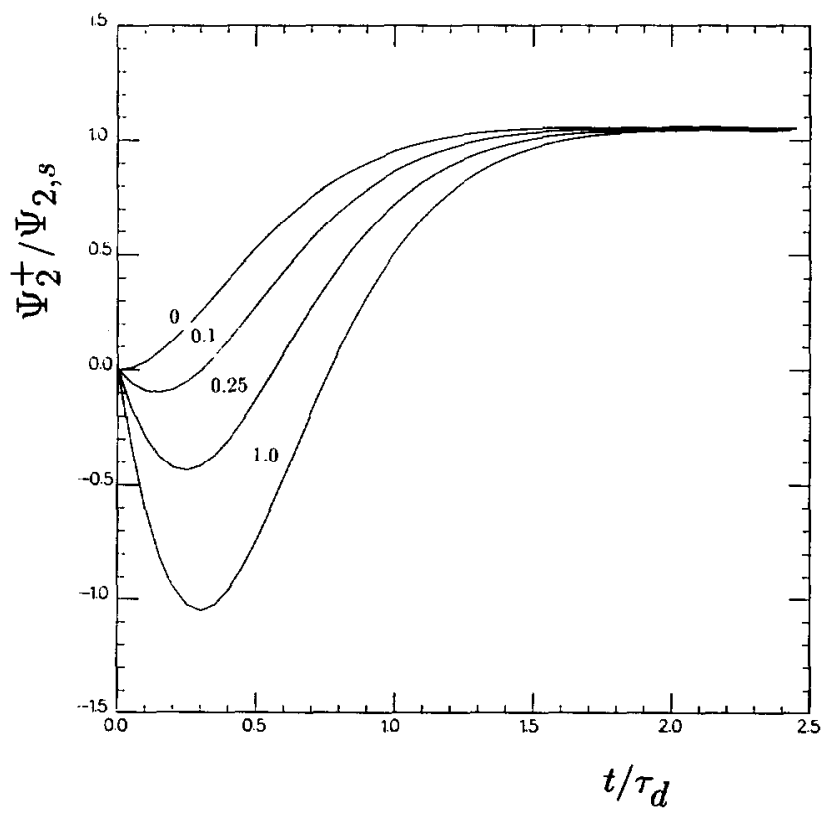

Fig. 3. Shear growth second normal stress coefficient $\Psi_{2}^{+}$normalized to the stationary values $\Psi_{2, s}$, as a function of $t / \tau_{d}$ at $\dot{\gamma}_{0} \tau_{d}=2$. The value of $\Delta$ used is indicated on the corresponding curve. 
found that the deviations from the Curtiss-Bird theory became more pronounced at constant $\Delta$ as $\dot{\gamma}_{0} \tau_{d}$ increases.

The first normal stress coefficient $\Psi_{1}^{+}$normalized to its stationary value $\Psi_{1, s}$ is plotted in Fig. 2. Again we notice a significant deviation from the Curtiss-Bird theory as $\Delta$ is small, and the deviation becomes more pronounced as $\dot{\gamma}_{0} \tau_{d}$ becomes larger. Finally in Fig. 3 we plotted the corresponding results for the second normal stress coefficient $\Psi_{2}^{+} / \Psi_{2, s}$. We notice that the unexpected decrease for small times, as reported by Curtiss and Bird becomes smaller as $\Delta$ decreases, and disappears completely as $\Delta \rightarrow 0$.

It should be remarked that the $\Delta=0$ limit theory is not equivalent to the Doi and Edwards theory. The onset phenomena as studied in this section show essential differences in the short and long time limit. Also, as will be pointed out in the next section, the viscometric functions for steady state shear flow differ considerably from those predicted by the Doi and Edwards theory at high shear rates. All these effects are due to the correlation related term in the constitutive eqn. (2.1).

\section{Steady state shear flow}

In steady state shear flow, the shear rate $\dot{\gamma}(t)$ is equal to some constant value $\dot{\gamma}_{0}$ for all times. The material functions $\eta\left(\dot{\gamma}_{0}\right), \Psi_{1}\left(\dot{\gamma}_{0}\right)$ and $\Psi_{2}\left(\dot{\gamma}_{0}\right)$ are defined as in (3.5-7), and are given explicitly by (3.15) upon taking the limit $t \rightarrow \infty$. We found that in all cases studied numerically the stationary state was approached very closely at least after about $25 \tau_{d}$, and we used this value as the upper limit in the integrations needed in (3.15). Before discussing these results, we present the material functions for small shear rates.

Using the small $\Gamma$ expressions (2.21-24) one may show after some calculation that, up to $\mathrm{O}\left(\left(\dot{\gamma}_{0} \tau_{d}\right)^{3}\right)$ the material functions can be written as

$$
\begin{aligned}
\frac{\eta\left(\dot{\gamma}_{0}\right)}{n N k T \tau_{d}}= & \frac{\pi^{2}}{60}\left[1+\frac{1}{3} \Delta\left(4-6 \Delta+4 \Delta^{2}-\Delta^{3}\right)\right] \\
& +\left(\dot{\gamma}_{0} \tau_{d}\right)^{2}\left[\frac{16}{35 \pi^{2}} \sum_{m=0}^{\infty} \frac{\alpha_{m}(\Delta)}{m^{8}}\right. \\
& \left.+\frac{16}{25} \sum_{m=0}^{\infty} \sum_{p=0}^{\infty} \frac{\beta_{m, p}(\Delta)}{m^{3} p^{3}}-\frac{16}{7 \pi^{2}} \sum_{m=0}^{\infty} \frac{1}{m^{8}}\right], \\
\frac{\Psi_{1}\left(\dot{\gamma}_{0}\right)}{n N k T \tau_{d}^{2}}= & \frac{\pi^{4}}{300}-\left(\dot{\gamma}_{0} \tau_{d}\right)^{2}\left[\frac{184}{21 \pi^{2}} \sum_{m=0}^{\infty} \frac{1}{m^{10}}\right. \\
& \left.-\frac{48}{21 \pi^{2}} \sum_{m=0}^{\infty} \frac{\alpha_{m}(\Delta)}{m^{10}}-\frac{32}{25} \sum_{m=0}^{\infty} \sum_{p=0}^{\infty} \frac{\beta_{m, p}(\Delta)}{m^{3} p^{5}}\right],
\end{aligned}
$$




$$
\begin{aligned}
\frac{\Psi_{2}\left(\dot{\gamma}_{0}\right)}{n N k T \tau_{d}^{2}}= & -\frac{32}{35 \pi^{2}}\left[\sum_{m=0}^{\infty} \frac{1}{m^{6}}-\frac{1}{2} \sum_{m=0}^{\infty} \frac{\alpha_{m}(\Delta)}{m^{6}}\right]+\left(\dot{\gamma}_{0} \tau_{d}\right)^{2}\left[\frac{768}{231 \pi^{2}} \sum_{m=0}^{\infty} \frac{1}{m^{10}}\right. \\
& \left.-\frac{384}{231 \pi^{2}} \sum_{m=0}^{\infty} \frac{\alpha_{m}(\Delta)}{m^{10}}-\frac{64}{175} \sum_{m=0}^{\infty} \sum_{p=0}^{\infty} \frac{\beta_{m, p}(\Delta)}{m^{3} p^{5}}\right]
\end{aligned}
$$

These are rather complicated expressions that reduce to the predictions obtained from the Curtiss-Bird theory with 'link tension coefficient' equal to $1 / 2$ as $\Delta=1$ [8]. The series in (4.1-3) containing $\alpha_{m}(\Delta)$ can be evaluated analytically, those containing $\beta_{m, p}(\Delta)$ cannot be expressed in closed form. As $\Delta=0$ we obtain expressions closely resembling those obtained by Doi and Edwards. The differences are all of second order in $\dot{\gamma}_{0} \tau_{d}$.

An interesting consequence of the present theory is that both positive and negative Weissenberg effects appear, depending on the value of $\Delta$. Consider $\Psi_{2}(0) / \Psi_{1}(0)$ which, as has been reported by Ramachandran et al. [17] takes on values between -0.1 and -0.3 in experimental situations. After some calculations, one may show that

$$
\sum_{m=0}^{\infty} \frac{\alpha_{m}(\Delta)}{m^{6}}=\frac{\pi^{6}}{960}\left[4 \Delta-5 \Delta^{2}+5 \Delta^{4}-4 \Delta^{5}+\Delta^{6}\right] .
$$

Hence

$$
\frac{\Psi_{2}(0)}{\Psi_{1}(0)}=-\frac{2}{7}\left[1-\frac{1}{2}\left[4 \Delta-5 \Delta^{2}+5 \Delta^{4}-4 \Delta^{5}+\Delta^{6}\right]\right]
$$

From this we notice that as $\Delta=0$ we obtain $-2 / 7$, consistent with the Doi and Edwards model using the independent alignment approximation to derive the one segment distribution function. As $\Delta=1$ we obtain $-1 / 7$ which is consistent with the Doi and Edwards model without the approximation [18-20] and the Curtiss-Bird model [8]. Since a positive Weissenberg effect appears at $\Psi_{2}(0) / \Psi_{1}(0)>-1 / 4$ [19], we see that for suitable choice of $\Delta$, we can obtain either a positive or negative Weissenberg effect.

We now present the numerical results obtained. In Fig. 4 we have plotted the shear viscosity $\eta / \eta_{0}$ as a function of $\dot{\gamma}_{0} \tau_{d}$. In this figure $\eta_{0}$ denotes $\eta(0)$. For low values of the shear rate we notice that varying $\Delta$ has hardly any effect on the shear viscosity. With increasing $\dot{\gamma}_{0} \tau_{d}$, provided $\dot{\gamma}_{0} \tau_{d}$ is small enough, the shear viscosity decreases and for small $\dot{\gamma}_{0} \tau_{d}$ it is accurately described by (4.1). However, as the shear rate is increased above a certain limit, which depends on $\Delta$, then, rather than decreasing monotonously as in the Curtiss-Bird model, the shear viscosity starts to increase. As $\Delta \rightarrow 1$ the value for $\dot{\gamma}_{0} \tau_{d}$ above which $\eta / \eta_{0}$ tends to increase tends itself to infinity. 


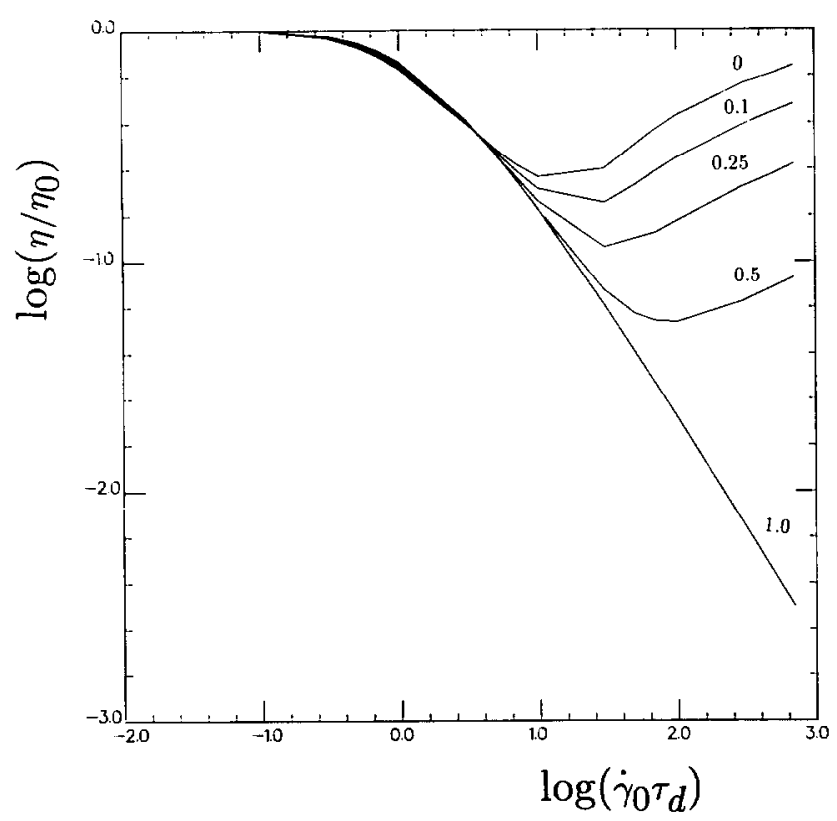

Fig. 4. Shear viscosity $\eta$ normalized to the zero shear rate value $\eta_{0}$ as a function of $\dot{\gamma}_{0} \tau_{d}$ at various values $\Delta$-values which are indicated on the corresponding lines.

Hence, this effect is absent in the Curtiss-Bird theory. In the limit $\dot{\gamma}_{0} \tau_{d} \rightarrow \infty$ the results indicate that

$\frac{\eta}{\eta_{0}} \approx\left(\dot{\gamma}_{0} \tau_{d}\right)^{\nu}$

with $\nu \approx 0.3$, independent of $\Delta$. In Fig. 5 , we have plotted the normalized shear stress as a function of shear rate. As in the previous figure the results do not depend sensitively on variations of $\Delta$ for low shear rates. However for high shear rates, rather than flattening out, as in the Curtiss-Bird model, the shear stress starts to increase again. The asymptotic behavior is again a power-law with exponent $\approx 1.3$. It is determined solely by the correlation related term in the constitutive equation (2.1). This unexpected behavior may be related to the slip-stick melt fracture phenomenon [21]. Contrary to what has been reported by Marucci and Grizutti [20], the shear viscosity at high shear rates increases. They predict such a phenomenon only in elongation experiments. Experimental evidence for this phenomenon has already been reported by Burow et al. [22] and Peterlin and Turner [23].

Finally in Fig. 6 we have plotted the first normal stress coefficient, normalized to $\Psi_{1,0} \equiv \Psi_{1}(0)$. Again we notice an essentially different behavior for large shear rates when compared to the Curtiss-Bird model. We also 


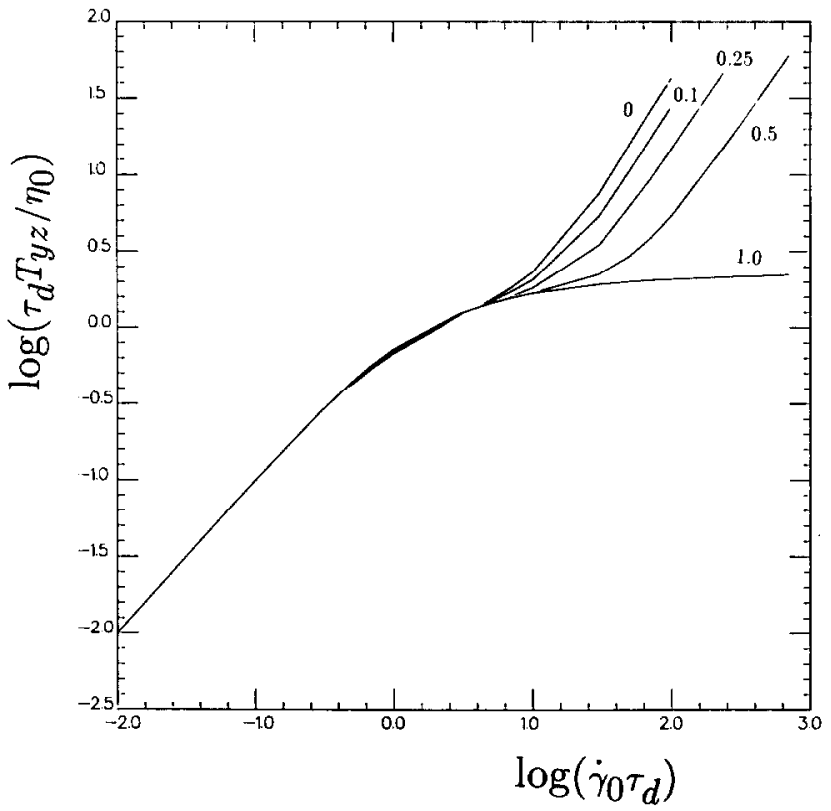

Fig. 5. Normalized shear stress $\tau_{d} T_{y z} / \eta_{0}$ as a function of $\dot{\gamma}_{0} \tau_{d}$ for various values of $\Delta$ which are indicated on the corresponding lines.

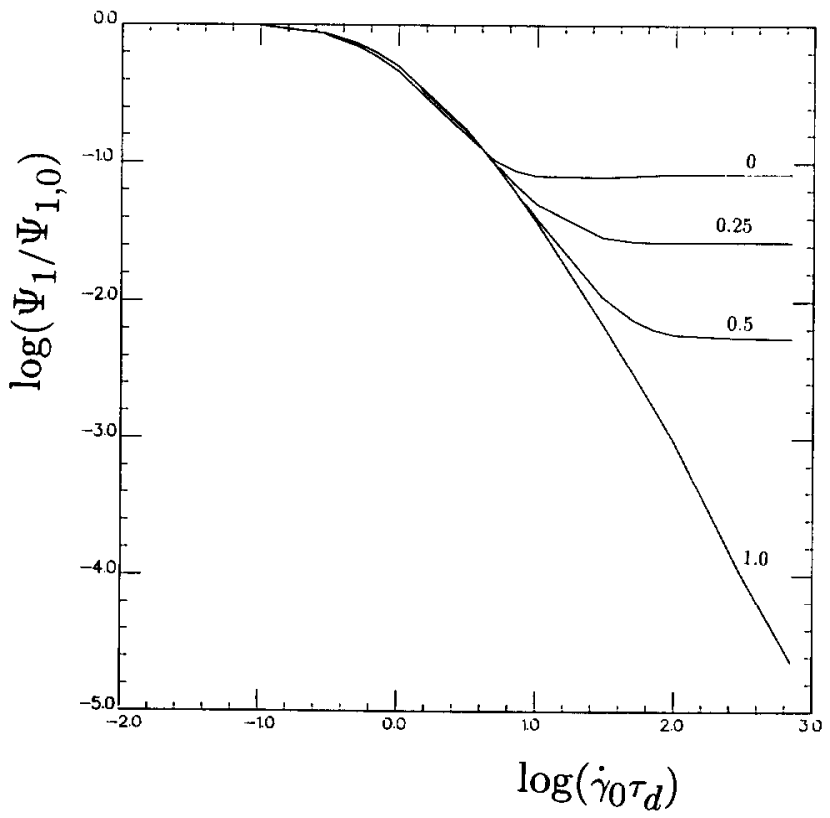

Fig. 6. First normal stress coefficient $\Psi_{1} / \Psi_{1,0}$ as a function of $\dot{\gamma}_{0} \tau_{d}$ for various values of $\Delta$ indicated at the corresponding lines. 
studied the second normal stress coefficient $\Psi_{2} / \Psi_{2,0}$. The results are qualitatively the same but were not determined as extensively in view of the large amount of computer time required.

In the next section we analyse for completeness the linear viscoelasticity as predicted by this model.

\section{Linear viscoelasticity; small amplitude oscillatory shear flow}

In small amplitude oscillatory shear flow the shear rate is defined by

$\dot{\gamma}(t)=\dot{\gamma}_{0} \cos (\omega t)$,

where $\dot{\gamma}_{0}$ is small. Hence we have

$\Gamma\left(t, t^{\prime}\right)=\frac{\dot{\gamma}_{0}}{\omega}\left[\sin (\omega t)-\sin \left(\omega t^{\prime}\right)\right]$.

Since $\dot{\gamma}_{0} / \omega$ is supposed to be small, we can use the small $\Gamma$ expressions $(2.21-2.24)$ in analysing the linear viscoelastic behavior. If we consider only terms linear in $\dot{\gamma}_{0}$ we can write the $y z$-component of the stress tensor in the form

$T_{y z}(t)=\dot{\gamma}_{0} \operatorname{Re}\left(G^{*}(\omega) e^{i \omega t}\right)$.

After some calculation one obtains, using (3.12)

$\frac{G^{*}(\omega)}{n N k T \tau_{d}}=\frac{8}{5 \pi^{2}} \sum_{m=0}^{\infty} \frac{1}{m^{2}\left(m^{2}+\mathrm{i} \omega \tau_{d}\right)}+\frac{\pi^{2}}{180} \Delta\left[4-6 \Delta+4 \Delta^{2}-\Delta^{3}\right]$.

Notice that the third term in the constitutive equation (2.1) does not contribute to $G^{*}(\omega)$ since it only contributes in second order and is not to be included in this analysis. The expression for $G^{*}(\omega)$ thus closely resembles that given by Curtiss and Bird in Ref. 8. We get full agreement if we associate the link tension coefficient $\epsilon$ introduced by Curtiss and Bird as follows

$\epsilon=\frac{1}{2} \Delta\left(4-6 \Delta+4 \Delta^{2}-\Delta^{3}\right)$

Of course, this identification is only valid as far as the linear viscoelasticity is concerned. For an illustration of the linear viscoelasticity, we refer the reader to Ref. 8.

\section{Acknowledgments}

The author is indebted to Dr. Ruud van Damme of the Department of Computer Science of Twente University for several useful discussions and to Drs. Robert J.J. Jongschaap for encouraging these calculations and helpful comments. 


\section{References}

1 B.J. Geurts and R.J.J. Jongschaap, J. Rheol., 32 (1988) 353.

2 R.J.J. Jongschaap, 2nd. Conf. of European Rheol. Prague, 1986.

3 R.J.J. Jongschaap and B.J. Geurts, Rolduc Polymer Meeting-2, 1987.

4 B.J. Geurts, J. Non-Newtonian Fluid Mech., 28 (1988) 319-322.

5 C.F. Curtiss and R.B. Bird, J. Chem. Phys., 74 (1981) 2016.

6 C.F. Curtiss and R.B. Bird, J. Chem. Phys., 74 (1981) 2026.

7 R.B. Bird, H.H. Saab and C.F. Curtiss, J. Phys. Chem., 86 (1982) 1102.

8 R.B. Bird, H.H. Saab and C.F. Curtiss, J. Chem. Phys., 77 (1982) 4747.

9 H.H. Saab, R.B. Bird and C.F. Curtiss, J. Chem. Phys., 77 (1982) 4759.

10 C.F. Curtiss and R.B. Bird, Physica, 118A (1983) 191.

11 P.G. deGennes, J. Chem. Phys., 55 (1971) 572.

12 M. Doi and S.F. Edwards, J. Chem. Soc. Faraday Trans., 74 (1978) 1789.

13 M. Doi and S.F. Edwards, J. Chem. Soc. Faraday Trans., 74 (1979) 1802.

14 M. Doi and S.F. Edwards, J. Chem. Soc. Faraday Trans., 74 (1979) 1818.

15 M. Doi and S.F. Edwards, J. Chem. Soc. Faraday Trans., 75 (1979) 38.

16 M. Doi and S.F. Edwards, The Theory of Polymer Dynamics, Clarendon Press, Oxford, 1986.

17 S. Ramachandran, H.W. Gao and E.B. Christiansen, J. Rheol., 25 (1981) 213.

18 G. Marucci, J. Non-Newtonian Fluid Mech., 21 (1986) 329.

19 G. Marucci and N. Grizutti, J. Non-Newtonian Fluid Mech., 21 (1986) 319.

20 G. Marucci and N. Grizutti, preprint, 1987.

21 Y.H. Lin, J. Non-Newtonian Fluid Mech., 23 (1987) 163.

22 S. Burow, A. Peterlin and D.T. Turner, Polym. Letters, 2 (1964) 67.

23 A. Peterlin and D.T. Turner, J. Chem. Phys., 30 (1963) 2315. 